\title{
Research data management (RDM)
}

Rosie Higman, Daniel Bangert, Sarah Jones

\section{Source}

Rosie Higman, Daniel Bangert, Sarah Jones. (2019). Three camps, one destination: the

intersections of research data management, FAIR and Open.

Research data management (RDM) can be defined as a set of practices to handle information collected and created during research. It is 'the compilation of many small practices that make your data easier to find, easier to understand, less likely to be lost, and more likely to be usable during a project or ten years later. ${ }^{[1]}$ These practices involve, but are not limited to, data management planning, documentation, organization, storage, dissemination and preservation. ${ }^{[2]}$ Effective RDM is an ongoing process which is structured and aligned with the research context and disciplinary practices.

\section{References}

1. ^ Kristin Briney. (2015). Data Management for Researchers. Pelagic Publishing Ltd.

2. ^ Louise Corti, Veerle Van den Eynden, Libby Bishop, Matthew Woollard. (2014). Managing and Sharing Research Data. SAGE Publications Limited. 